\title{
Combined Proctectomy and Hepatectomy for Metastatic Rectal Cancer: Safe for the Fit and Able-But for Everyone?
}

\author{
James R. Barrett, MD, and Daniel E. Abbott, MD \\ Division of Surgical Oncology, Department of Surgery, Clinical Science Center, University of Wisconsin, Madison, WI
}

This month's issue of Annals of Surgical Oncology features a publication by Concors et al. illustrating contemporary variability in outcomes for patients undergoing combined hepatectomy and proctectomy (C-PH) for rectal cancer. ${ }^{1}$ Importantly, this study provides increasing evidence that C-PH at high-volume centers is safe and can be primarily utilized for patients with synchronous hepatic metastasis from rectal cancer; these data are critical for patient counseling and surgical decision making. Furthermore, the authors are able to identify a subset of patients, particularly those who are older and more frail, in whom C-PH should be approached with extreme caution. This clinically relevant, multi-institutional investigation capitalizes on the strengths of the National Cancer Data Base (NCDB) in reporting real-world utilization and outcomes in the US.

Colorectal cancer with synchronous liver metastases comprises at least $15 \%$ of colorectal cancer presentations. ${ }^{2}$ While surgical resection offers the only opportunity for long-term survival, ${ }^{3}$ only $10-15 \%$ of patients with hepatic metastasis are eligible for resection. ${ }^{4}$ Previous investigations into the safety of C-PH include a 10-year cohort study of 1430 colorectal cancer patients from the New York State SPARCS database, which demonstrated that simultaneous resection, as opposed to staged procedures, was performed in the majority of eligible cases $(76 \%)$ and was associated with equivalent short-term perioperative outcomes. This approach was associated with a significantly shorter length of stay and significantly decreased healthcare utilization in the simultaneous resection cohort. ${ }^{5}$

(C) Society of Surgical Oncology 2019

First Received: 9 May 2019;

Published Online: 12 June 2019

D. E. Abbott, MD

e-mail: abbott@surgery.wisc.edu
However, C-PH continues to be a somewhat controversial practice, with variable clinical outcomes demonstrated in the literature. ${ }^{6,7}$ Notably, a prospective pilot study (RESECT) is currently underway, and was designed to assess 90-day postoperative complication rate, postoperative 90-day mortality, quality of life at 3 months, and healthcare utilization in patients diagnosed with synchronous colorectal liver metastasis undergoing a simultaneous colorectal and liver resection (including major liver resections). ${ }^{8}$

As with all registry-based series, there are challenges in interpreting and generalizing the results of the current study by Concors et al. A noteworthy limitation is the inability to precisely determine the extent of liver surgery-not only whether hepatic surgery was the 'other' operation performed at the time of proctectomy but also the extent of hepatectomy. While variability between right or left hepatectomy is less pronounced, there can be significant differences between 'partial hepatectomy' (e.g. a posterior sectorectomy versus a wedge of segment 3 ). The authors certainly acknowledge this limitation, although it cannot be understated - the extent of hepatectomy is crucial to perioperative outcomes in this scenario. Similarly, the extent of pelvic surgery is difficult to discern from NCDB data, with the inability to determine the location of anastomosis and/or whether a diverting loop ileostomy was performed.

Special attention must also be paid to the outcome variables most germane to this type of analysis. Appropriately, the authors have primarily focused on perioperative mortality, arguably the most critical endpoint that exists. Certainly though, there are other endpoints that impact patient quality of life, including pelvic and/or perihepatic abscess, anastomotic leak, long-term bowel function, and/or postoperative hepatic insufficiency. These complications are not all created equal, and severity stratification is important. It is possible, if not very likely, that 
patients would preferentially endure complications and multiple surgeries in favor of a lower mortality rate. Conversely, one could envision that a pelvic abscess or a leak (especially if the patient was diverted) may be preferable to two operations.

In an era of increasing cost consciousness, it is important to consider the fiscal consequences of simultaneous versus staged resection for these patients. As healthcare costs in the US continue to rise, with projections estimating that healthcare expenditure will consume $25 \%$ of the gross domestic product in 2025 , further comparative and costeffectiveness investigations are necessary, while ensuring that we are not compromising patient safety, quality of healthcare delivery, or long-term outcomes. ${ }^{9}$ Previous work in this patient population has demonstrated a potential cost savings of approximately $17 \%$ for patients undergoing simultaneous resection, without detriment in short-term outcomes. ${ }^{10}$ Clearly, if perioperative outcomes and oncologic efficacy are equivalent, all stakeholders would agree that simultaneous resection is the favored approach. Although the authors of the current study did not have the tools at their disposal to perform a resource utilization analysis, we look forward to the results of the previously mentioned RESECT study as it will incorporate both clinical outcomes and healthcare utilization.

Most importantly, this study has illuminated significant demographic and socioeconomic disparities in the delivery of C-PH to patients who could potentially benefit from this approach. This discovery should stimulate health systemlevel discussion of how providers can refer appropriate patients for C-PH if they themselves do not have the optimal clinical expertise and/or resources to perform $\mathrm{C}-\mathrm{PH}$. In particular, these data should be strongly considered in the decision to perform C-PH in patients over 70 years of age or with high-risk features, and should certainly be used to help all patients make an informed decision about their care. Ultimately, Concors et al. have provided the surgical oncology community with a highquality study that will help inform the care of complex, high-risk rectal cancer patients in a safe and cost-effective manner, with the hope that it will inform policy changes aimed at providing this standard (when safe) to all fit and able rectal cancer patients.

\section{REFERENCES}

1. Concors SJ, Vining CM, et al. Combined proctectomy and hepatectomy for metastatic rectal cancer should be undertaken with caution: results of a national cohort study annals of surgical oncology. In Press

2. Manfredi S, Lepage C, Hatem C, Coatmeur O, Faivre J, Bouvier AM. Epidemiology and management of liver metastases from colorectal cancer. Ann Surg. 2006;244(2):254-259.

3. Robertson DJ, Stukel TA, Gottlieb DJ, Sutherland JM, Fisher ES. Survival after hepatic resection of colorectal cancer metastases: a national experience. Cancer. 2009;115(4):752-759.

4. Schlag PM, Benhidjeb T, Stroszczynski C. Resection and local therapy for liver metastases. Best Pract Res Clin Gastroenterol. 2002;16(2):299-317.

5. Abelson JS, Michelassi F, Sun T, et al. Simultaneous resection for synchronous colorectal liver metastasis: the new standard of care? J Gastrointest Surg. 2017;21(6):975-982.

6. Silberhumer GR, Paty PB, Temple LK, et al. Simultaneous resection for rectal cancer with synchronous liver metastasis is a safe procedure. Am J Surg. 2015;209(6):935-942.

7. Hamed OH, Bhayani NH, Ortenzi G, et al. Simultaneous colorectal and hepatic procedures for colorectal cancer result in increased morbidity but equivalent mortality compared with colorectal or hepatic procedures alone: outcomes from the National Surgical Quality Improvement Program. HPB (Oxford). 2013;15(9):695-702.

8. Pablo ES, Gafni A, Parpia S, Ruo L, Simunovic M, Meyers BM, Reiter H, et al. Simultaneous resection of colorectal cancer with synchronous liver metastases (RESECT), a pilot study. Int J Surg Protoc. 2018;8:1-6

9. Munoz E, Munoz W 3rd, Wise L. National and surgical health care expenditures, 2005-2025. Ann Surg. 2010;251(2):195-200.

10. Abbott DE, Cantor SB, Hu CY, et al. Optimizing clinical and economic outcomes of surgical therapy for patients with colorectal cancer and synchronous liver metastases. J Am Coll Surg. 2012;215(2):262-270.

Publisher's Note Springer Nature remains neutral with regard to jurisdictional claims in published maps and institutional affiliations. 